\title{
COVID-19 pandemic: effects and evidence-based recommendations for otolaryngology and head and neck surgery practice
}

\author{
Luiz P. Kowalski et al. ${ }^{1}$ \\ ${ }^{1}$ Head and Neck Surgery Department, University of Sao Paulo Medical School and \\ Department of Head and Neck Surgery and Otorhinolaryngology, A C Camargo Cancer \\ Center, Sao Paulo, Brazil
}

April 28, 2020

\begin{abstract}
The 2019 novel coronavirus disease (COVID-19) is a highly contagious zoonosis produced by SARS-CoV-2 that is spread human-to-human by respiratory secretions. It was declared by the WHO as a public health emergency. The most susceptible populations, needing mechanical ventilation, are the elderly and people with associated comorbidities.

There is an important risk of contagion for anesthetists, dentists, head and neck surgeons, maxillofacial surgeons, ophthalmologists and otolaryngologists. Health workers represent between $3.8 \%$ to $20 \%$ of the infected population; some $15 \%$ will develop severe complaints and among them, many will lose their lives. A large number of patients do not have overt signs and symptoms (fever/respiratory), yet pose a real risk to surgeons (who should know this fact and must therefore apply respiratory protective strategies for all patients they encounter).

All interventions that have the potential to aerosolize aerodigestive secretions should be avoided or used only when mandatory. Health workers who are: pregnant, over 55-65 years of age, with a history of chronic diseases (uncontrolled hypertension, diabetes mellitus, chronic obstructive pulmonary diseases and all clinical scenarios where immunosuppression is feasible, including that induced to treat chronic inflammatory conditions and organ transplants) should avoid the clinical attention of a potentially infected patient. Healthcare facilities should prioritize urgent and emergency visits and procedures until the present condition stabilizes; truly elective care should cease and discussed on a case-by-case basis for cancer patients.

For those who are working with COVID-19 infected patients' isolation is compulsory in the following settings: a) unprotected close contact with COVID-19 pneumonia patients: b) onset of fever, cough, shortness of breath and other symptoms (gastrointestinal complaints, anosmia and dysgeusia have been reported in a minority of cases).

For any care or intervention in the upper aerodigestive tract region, irrespective of the setting and a confirmed diagnosis (e.g.; rhinoscopy or flexible laryngoscopy in the outpatient setting and tracheostomy or rigid endoscopy under anesthesia) it is strongly recommended that all healthcare personnel wear personal protective equipment (PPE) such as N95, gown, cap, eye protection and gloves.

The procedures described are essential in trying to maintain safety of healthcare workers during COVID-19 pandemic. In particular, otolaryngologists, head and neck, and maxillofacial surgeons are per se exposed to the greatest risk of infection while caring for COVID-19 positive subjects, and their protection should be considered a priority in the present circumstances.
\end{abstract}

\section{Full author list}

Luiz P. Kowalski, MD, $\mathrm{PhD}^{1}$, Alvaro Sanabria, MD, $\mathrm{PhD}^{2}$, John A. Ridge, MD, PhD, FACS ${ }^{3}$, Wai Tong Ng, MBChB, MD, FRCR, FHKCR, FHKAM ${ }^{4}$, Remco de Bree, MD, PhD ${ }^{5}$, Alessandra Rinaldo, MD, FRCSEd ad hominem, FRCS (Eng, Ir) ad eundem, FRCSGlasg, FACS ${ }^{6}$, Robert P. Takes, MD, PhD ${ }^{7}$, Antti A. Mäkitie, MD, $\mathrm{PhD}^{8}$, Andre L. Carvalho, $\mathrm{MD}, \mathrm{PhD}, \mathrm{MPH}^{9}$, Carol R. Bradford, $\mathrm{MD}^{10}$, Vinidh Paleri, MS, FRCS (ORL-HNS) ${ }^{11}$, Dana M. Hartl, $\mathrm{MD}, \mathrm{PhD}^{12}$, Vincent Vander Poorten, MD, $\mathrm{PhD}^{13}$, Iain J. Nixon, MD,MBChB,FRCS (ORL-HNS)PhD ${ }^{14}$, Cesare Piazza, $\mathrm{MD}^{15}$, Peter Lacy, MD, FRCSI ${ }^{16}$, Juan P. Rodrigo, $\mathrm{MD}, \mathrm{PhD}^{17}$, Orlando Guntinas-Lichius, $\mathrm{MD}^{18}$, William M. Mendenhall, $\mathrm{MD}^{19}$, Anil D'Cruz, MS, DNB, FRCS (Hon) ${ }^{20}$, Anne W.M. Lee, MBBS, MD, FRCR, FHKCR, FHKAM ${ }^{21}$, Alfio Ferlito, MD, 
DLO, DPath,FRCSEd ad hominem, FRCS (Eng, Glasg, Ir) ad eundem,FDSRCS ad eundem, FACS, FHKCORL, FRCPath, FASCP, IFCAP 22

${ }^{1}$ Head and Neck Surgery Department, University of Sao Paulo Medical School and Department of Head and Neck Surgery and Otorhinolaryngology, A C Camargo Cancer Center, Sao Paulo, Brazil.

${ }^{2}$ Department of Surgery, School of Medicine, Universidad de Antioquia, Centro de Excelencia en Cirugia de Cabeza y Cuello-CEXCA, Medellin, Colombia.

${ }^{3}$ Head and Neck Surgery Section, Department of Surgical Oncology, Fox Chase Cancer Center, Philadelphia, PA, USA.

${ }^{4}$ Department of Clinical Oncology, Pamela Youde Nethersole Eastern Hospital, Hong Kong, China

${ }^{5}$ Department of Head and Neck Surgical Oncology, UMC Utrecht Cancer Center, University Medical Center Utrecht, Utrecht, The Netherlands

${ }^{6}$ University of Udine School of Medicine, Udine, Italy

${ }^{7}$ Department of Otolaryngology-Head and Neck Surgery, Radboud University Medical Center, Nijmegen, The Netherlands

${ }^{8}$ Department of Otorhinolaryngology, Head and Neck Surgery, University of Helsinki and Helsinki University Hospital, Finland; Research Program in Systems Oncology, Faculty of Medicine, University of Helsinki, Helsinki, Finland; Division of Ear, Nose and Throat Diseases, Department of Clinical Sciences, Intervention and Technology, Karolinska Institute and Karolinska Hospital, Stockholm, Sweden

${ }^{9}$ Screening Group. International Agency for Research in Cancer. IARC, Lyon, France

${ }^{10}$ Department of Otolaryngology, University of Michigan, Ann Arbor, USA

${ }^{11}$ Head and Neck Unit, The Royal Marsden Hospital, London, UK

${ }^{12}$ Departments of Otolaryngology Head and Neck Surgery, Institut Gustave Roussy and University Paris-Sud, Villejuif Cedex, France

${ }^{13}$ Department of Oncology, section Head and Neck Oncology, KU Leuven, and Otorhinolaryngology, Head and Neck Surgery, University Hospitals Leuven, Leuven Cancer Institute, Leuven, Belgium

${ }^{14}$ Department of Otolaryngology, Head and Neck Surgery, NHS Lothian, University of Edinburgh, UK

${ }^{15}$ Department of Otorhinolaryngology, Maxillofacial, and Thyroid Surgery, Fondazione IRCCS, National Cancer Institute of Milan, University of Milan, Milan, Italy

${ }^{16}$ Department of Otolaryngology, Beaumont Hospital, Dublin, Ireland.

${ }^{17}$ Servicio de Otorrinolaringología, Hospital Universitario Central de Asturias. Instituto Universitario de Oncología del Principado de Asturias, Universidad de Oviedo. Oviedo, Spain

${ }^{18}$ Department of Otorhinolaryngology, Institute of Phoniatry/Pedaudiology, Jena University Hospital, Jena, Germany

${ }^{19}$ Department of Radiation Oncology, University of Florida, Gainesville, FL, USA

${ }^{20}$ Head Neck Services, Tata Memorial Hospital, Parel, Mumbai, 400012, India

${ }^{21}$ Department of Clinical Oncology, The University of Hong Kong, Hong Kong, China

${ }^{22}$ Coordinator of the International Head and Neck Scientific Group. Udine, Italy

This article was written by members and invitees of the International Head and Neck Scientific Group (www.IHNSG.com). 
Disclaimer: Andre L. Carvalho is responsible for his views expressed in this article and they do not necessarily represent the decisions, policy or views of the International Agency for Research on Cancer / World Health Organization.

\section{The facts}

The 2019 novel coronavirus disease (COVID-19) pandemic started in December 2019 in the city of Wuhan, Hubei province in China. It is a highly contagious zoonosis (with a reproductive number of 2.8, which means that under preexisting conditions one case generates 2.8 new cases) produced by a beta coronavirus (SARSCoV-2) that is spread human-to-human largely by respiratory secretions and occasionally by feces. ${ }^{1}{ }^{2}$ Over a few weeks the disease spread to other Asian countries, to Europe, to the Americas and finally across the world demonstrating a rapid doubling time (6.4 days) and an asymptomatic but highly infectious prodrome. ${ }^{2,}{ }^{3}$ On January 20, 2020 it was declared by the WHO to represent a public health emergency. According to the Johns Hopkins Dashboard, as of March 20, 2020, 166 countries and 274,180 patients had been confirmed to be infected, 11,375 have died and 87,991 have recovered. The infections occurred predominantly (87\%) in people of 30-79 years-old. 4 Most infections (81\%) are asymptomatic or produce only mild symptoms, whereas $15 \%$ occur in severe form that has required hospitalization. Some 3-4\% benefit from respiratory support in an intensive care unit (ICU). ${ }^{4}$ The death rate has been calculated between $0.39-4 \%$, but this depends upon patient age and is much higher in those older than 70 years. ${ }^{1,} 4$ The most likely population to require mechanical ventilation are the elderly and people with associated comorbidities (in particular cardiovascular disease and hypertension, followed by diabetes mellitus) with a predicted mortality of around 15-49\%.4

Transmission is mainly produced by symptomatic patients, but it has been reported that even asymptomatic individuals and those in the incubation period (which can last longer than 14 days), can also be a source of occult transmission. ${ }^{4}$ Swab PCR results for most asymptomatic patients turn negative in about 3 days, while symptomatic patients typically have detectable virus for $12-20$ days. ${ }^{3,5}$ There is still little information about the transmission during the recovering phase. ${ }^{1}$

Most patients treated by head and neck surgeons have cancer, the direct role of SARS-CoV-2 infection in their outcomes is unknown. To date, there is no clear evidence that cancer patients have an increased risk of infection or severe disease, beyond the immunosuppression caused by the malignancy itself. ${ }^{6}$ However, cancer patients have experienced a higher risk of death due to the limitations of access imposed by social distancing and the shortage of operating rooms and ICU beds. ${ }^{7}$

\section{The risk for otolaryngologists, head and neck, and maxillofacial surgeons}

The first reported physician fatality related to COVID-19 in Wuhan, China, was that of an otolaryngology physician on January $25,2020 .{ }^{8}$ In Wuhan epidemic statistics, health workers represented $3.8 \%$ of the infected, $14.8 \%$ had severe disease and the overall mortality rate was of $0.6 \% .^{1,4,9}$ On the other hand, in Italy, $20 \%$ of responding healthcare workers were infected, and some have died. ${ }^{10}$ People in contact with symptomatic patients, such as health personnel, are the most susceptible to infection. The most commonly infected healthcare personnel worked in general wards. ${ }^{11}$

Human-to-human spread occurs through respiratory secretions (although fecal-oral spread has also been confirmed) so healthcare personnel that manage patients with diseases of the aerodigestive tract (dentists, otolaryngologists, head and neck surgeons, gastroenterologists, pneumonologists, respiratory therapists, speech therapists, and infectious disease physicians) or ophthalmologists are the most susceptible healthcare workers to become infected (risk ratio of 2.13). ${ }^{1,12,13}$ Therefore it was rapidly recognized that there is a particular need for protective measures in these professional groups. ${ }^{14}$

In cases of COVID-19 patients with known respiratory disease, protective measures are usually followed by surgeons. However, a significant number of patients do not have fever nor respiratory symptoms (13$30 \%$ ), so surgeons should apply respiratory protective strategies for all patients. This is especially true in tropical countries, were symptoms can simulate other viral infectious diseases such as dengue. ${ }^{15}$ The classical 
symptoms of the infection are fever, dry cough and shortness of breath. The syndrome rarely resembles a classical "cold" or with a runny nose that helps distinguish it from the common viral flu. Nonetheless, precautions must be taken for all patients with flu-like symptoms.

Recent reports from sites around the world have shown that anosmia and dysgeusia are significant symptoms associated with the COVID-19 pandemic. Anosmia, in particular, has been seen in patients ultimately testing positive for the coronavirus with no other symptoms. For this reason, the American Academy of Otolaryngology (AAO-HNSF) has proposed that these symptoms be added to the list of screening tools for possible COVID-19 infection. (https://www.entnet.org/content/aao-hns-anosmia-hyposmia-and-dysgeusia-symptomscoronavirus-disease)

All procedures that have the potential to aerosolize aerodigestive secretions, such as nasolaryngoscopy, endotracheal intubation, non-invasive ventilation, transnasal endoscopic surgery and high-speed handpieces or ultrasonic instruments, increase the risk of infection and should be avoided or employed only when mandatory. ${ }^{1,16}$ There is no information regarding any potential risk for electrocautery smoke or transoral laser resection generated smoke but it would be reasonable to take appropriate precautions in these settings too.

Due to the characteristics of the virus, the standard protective measures of daily workflow do not prevent the infection, and specific masks (N-95 or FFP2 or higher) or powered air-purifying respirator (PAPR), other PPE and dedicated sterilization measures should be implemented to avoid the infection. ${ }^{17,} 18$ However, one case series report that none of 41 health workers that had contact with aerosolized secretions of COVID-19 positive patients and employed standard PPE, developed an infection. It suggests that the rate of infection, when standard measures are employed, is considerably lower than when they are not used or are used improperly. ${ }^{12,19}$ One of the more important reasons to explaining healthcare workers infection may be related to the lack of PPE and education about its correct use. ${ }^{9}$ It has been suggested that standard measures properly followed are more successful than the quick implementation of complex protection strategies. ${ }^{12,} 20$

Pregnancy, age over 55 years, some chronic diseases (chronic hepatitis, renal diseases, diabetes mellitus, autoimmune diseases and cancer) represent risk factors for developing severe acute respiratory distress syndrome (ARDS); affected health workers should not take care for infected patients. ${ }^{21}$ There is no information about intrauterine or transplacental transmission to the newborn. ${ }^{22,} 23$

The disease also imposes a physical, mental and emotional burden to healthcare workers. ${ }^{24}$ Most physicians caring for infected patients and for patients considered "suspicious" for infection become more quickly exhausted. There is also a specific risk associated with "health anxiety"; a phenomenon defined as "anxiety that occurs when perceived sensations of changes are interpreted as symptoms of being ill ." 25 This occurs commonly in daily life, but in times of infectious diseases outbreaks, this phenomenon can produce detrimental effects. Today, (accurate, but often also incorrect) information about the clinical course, rate of complications and mortality of COVID-19 is incessantly emphasized and widespread through media and social networks. This increases the frequency and severity of health anxiety, characterized by catastrophic misinterpretation of sensations, wrong beliefs about the disease's consequences and dysfunctional coping. ${ }^{25,}{ }^{26}$ As more information is received and more ensues, excess anxiety results in a loss of the ability to make rational decisions. Specifically for surgeons, this situation could have two polar effects: firstly, some people may see doctors as a source of contagion and avoid them (and thus, medical assistance); secondly, other people may see them as source of security and visit them repeatedly, putting a further burden on strained health services. Both situations increase the risk for surgeons: in the first case, they can be threatened in public areas and in the second their capacity to help may be overwhelmed. An infection-related xenophobia is also a potential risk for foreign surgeon's practice. ${ }^{11,27}$

Finally, the impact of financial concerns of healthcare workers on their wellbeing and performance should not be underestimated.

\section{Recommendations for clinical and endoscopic examination}


The risk of contamination is very high in upper respiratory examinations. In Chinese patients, SARS-Cov-2 was detected in $63 \%$ of nasopharyngeal swabs, in $46 \%$ of the fiberoptic bronchoscopic brush biopsies and in $93 \%$ of bronchoalveolar lavage fluid specimens. ${ }^{28}$ Higher viral loads were detected after symptoms onset of COVID-19, with higher viral load in the nose than in the throat. ${ }^{29}$ In spite of that, recommendations for clinical and endoscopic examination can be very controversial because adequate PPE to all staff involved in patient care cannot be available everywhere. For sure, at least "WHO infection prevention and control (IPC) standards" should be in place at international level. Disruption of supply chains and depletion of stock of PPE can drive anxiety in health professionals. ${ }^{30}$

The Working Group of 2019 Novel Coronavirus, Peking Union Medical College Hospital, ${ }^{21}$ criteria for the selection of front-line medical personnel included passing physical examinations and professional training for COVID-19. Candidates were excluded if they were pregnant, aged over 55 years, had history of chronic diseases such as chronic hepatitis, renal diseases, diabetes mellitus, autoimmune diseases, and tumors. All individuals with acute fever were also excluded. For those who are working with COVID-19 suspicious or infected patients, isolation or observation was compulsory in the following conditions: a) those with close unprotected contact with COVID-19 pneumonia patients (health workers should be relatively isolated, "avoiding walking around and extensive contact with others"); b) onset of fever, cough, shortness of breath and other symptoms (medical personnel should be isolated immediately and receive appropriate care); c) when work in the COVID-19 infection ward is finished, nasopharyngeal or oropharyngeal swabs for COVID19 and a full blood count should be carried out. Those who have abnormal test results should undergo strict isolation and observation; while others will be generally isolated for observation and resume work after one week. However, there are no consensus on these recommendations as to how long COVID-19-positive healthcare professionals should refrain from patient care. Viral secretion was found in hospitalized Chinese patients who recovered to last from 8 to 37 days with a median of 20 days. ${ }^{5}$

During the COVID-19 pandemic status, effective biosafety precautions must be implemented in all clinics and hospitals because most infected patients are not symptomatic and may have been examined only with inadequate protection. Medical and dental examinations and procedures can produce aerosols. Even subsequent patients in the same room or suite are at elevated risk. ${ }^{1} 31,32$. Consequently, healthcare professionals are at high risk of contamination.

Aiming to preserve and ensure staff and patient safety, the USA Center for Diseases Control (CDC) recommends that healthcare facilities cease elective care and restrict their activities to providing urgent and emergency visits and procedures for several weeks. All elective and non-time sensitive, non-urgent surgical procedures and admissions must be rescheduled as necessary. ${ }^{33}$ In other parts of the world, like Hong Kong, and, and most recently, Italy, France and Belgium, there has also been a reduction in elective clinics and operations, with the aim of prioritizing the use of personnel and available facilities to the diagnostic and therapeutic pathways of COVID-19 management. However, most oncologic interventions are still proceeding in the belief that cancer surgery is time-sensitive. It would be prudent to confirm that the patient is SARS-CoV-2 free through swab testing. This recommendation must change when the situation becomes critical and the whole healthcare facility is under strain from COVID-19 patients. ${ }^{8}$ This may ensue even in more-developed countries.

Working in the epicenter of the current pandemic,${ }^{1} 169$ staff workers involved in a dental emergency unit at the School and Hospital of Stomatology, Wuhan, China, have treated $>700$ patients with emergent dental care since January 24 under using adequate protection measures. Since February 3, they have also provided consultations to $>1,600$ patients on an online platform. No COVID-19 infection has been reported among the staff, confirming the effectiveness of the established infection control measures in COVID-19 prevention within dental settings. They established triage stations to measure and record the temperature of all staff and patients. Patients and accompanying persons were provided with medical masks and temperature measurement once they entered the hospital. All the dentists undertook strict personal protection measures and avoided techniques that could produce aerosols. Rubber dams and high-volume saliva ejectors were used to minimize aerosol or spatter during the surgical procedures. Face shields and goggles were considered essential 
with use of drilling with water spray. A 4-handed technique and saliva ejectors were considered beneficial.

\section{Risk reduction using remote triaging for new referrals and follow ups}

To break the chain of transmission, several healthcare jurisdictions are recommending reduced footfall in hospitals. Thus, structured telephone triaging can be used to separate low-risk from high-risk patients, with the latter cohort being seen sooner. Using one such risk calculator generated iteratively from over 10,000 patients in the UK, the risk of missing cancer in low-risk patients who do not undergo a face-toface consultation is under $2 \%{ }^{34}$ Such patients can be reassessed at a later time when the acute pandemic associated issues are resolved. This approach has been supported by ENTUK, the British Association of Otolaryngology-Head and Neck Surgery.

Similarly, it has been well recognized that the detection of recurrent cancers is low in the population of asymptomatic patients after treatment. These patients need support with functional problems. They should be offered remote consultation; only those with new symptoms should be offered face-to-face consultation for the duration of the pandemic. ${ }^{35}$

\section{Indications and care during intubation and tracheostomy}

Head and neck surgeons, otolaryngologists, maxillofacial surgeons and anesthetists are at high risk of contamination and infection by SARS-CoV-2 when assisting patients with tracheostomy or performing a rhinoscopy or a laryngoscopy because of the generation of aerosols. Fortunately, tracheostomy is unlikely to be required for the majority of patients. All patients must be examined by a physician wearing PPE such as N95 or FFP2 mask or PAPR, gown, cap, eye protection and gloves. ${ }^{8,} 28$ For a tracheostomy, all staff must keep PPE with a powered air-purifying respirator (PAPR) throughout the procedure. ${ }^{11}$

The Royal College of Surgeons has posted guidance for surgical tracheostomy during the COVID-19 pandemic (https://www.entuk.org/tracheostomy-guidance-during-covid-19-pandemic), with advice as to decreasing the risk of aerosolization: stop ventilation while the tracheostomy window is being performed, and only resume ventilation when the cuff of the tracheostomy tube has been inflated.

Zuo and co-workers presented the Chinese Society of Anesthesiology Task Force on Airway Management recommendations for proper practice of tracheal intubation by frontline anesthesiologists and critical care physicians in critically ill patients with COVID-19 disease. ${ }^{36}$ Intubation is considered a high-risk procedure due to the physician exposure to secretions, blood, droplets and aerosols and should be indicated only for patients with severe respiratory distress or hypoxemia after standard oxygen therapy. It should be undertaken in an airborne isolation room and enhanced droplet/airborne PPE should be applied to all the healthcare workers, including N95 masks, hair cover, protective coverall, gown, gloves, face shields and goggles (prepared for anti-fog) and shoe covers. If available, a protective head hood or PAPR should be used. Whenever possible, it should be performed by an experienced anesthesiologist with assistance of another physician. Airway assessment before intervention is regarded as crucial. All airway management tools must be disposable and available including a videolaryngoscope with disposable blades, and devices for needle or scalpel cricothyroidotomy. According to Aminnejad et al. ${ }^{37}$ and Yang et al. ${ }^{38}$ the administration of intravenous lidocaine prior to tracheal extubation can reduce coughing without side-effects, and it can be recommended also for intubation aiming to reduce the risk for the physician who is doing the procedure. Several specific recommendations are also afforded for an anticipated difficult airway. The endotracheal tube must be secure and then the patient is put in mechanical ventilation. All devices must be collected in double-sealed bags and proper disinfection implemented during disposal. All equipment and environment surfaces must be cleaned and disinfected. ${ }^{36}$

Akin to the physicians at high risk, allied health and nursing professionals who care for patients with head and neck diseases continue to be at high risk. Problematic settings include postoperative tracheostomy care and valve changes for laryngectomees. These personnel should adhere to the same PPE recommendations as for other professional groups involved in airway interventions.

\section{Operating on patients with COVID-19}


In preparation for the COVID-19 pandemic, Wong et al. ${ }^{39}$ reviewed operating room (OR) outbreak response measures. Several recommendations can be implemented worldwide, others must be adapted according to the resource availability. An OR with a negative pressure environment is ideal to reduce dissemination of the virus. A high frequency of air changes (25 per hour) reduces viral load within the OR. Separate ORs can be designated for surgery in patients suspected or confirmed to have COVID-19. Each OR must have its own ventilation system with an integrated high-efficiency particulate air (HEPA) filter. These ORs should be separated from the main OR complex to reduce the risk of contaminating other ORs. Traffic and flow of contaminated air can be minimized by locking all doors to the OR during surgery, with only one possible route for entry and exit. It is considered important to have a program for the use of PPE. All healthcare personnel must be trained in the use of PPE. Postoperative visits must be suspended and replaced by phone calls to reduce movement of staff around the hospital.

Ti et al. ${ }^{40}$ recommend that an OR with a negative pressure environment with separate access must be used to operate suspected or confirmed cases of COVID-19 infection. They also advocate that the same room and the same anesthesia machine should be used for all COVID-19 patients during the epidemic. During the surgical procedure a runner wearing PPE is stationed outside the OR in case other drugs or equipment are needed.

The current recommendations of the American Academy of Otolaryngology-Head and Neck Surgery is that all elective surgical treatments should be rescheduled, but it is unavoidable to provide surgical care to patients with time-sensitive, urgent or emergent medical conditions (https://www.entnet.org/content/coronavirusdisease-2019-resources). ${ }^{41}$ Many of these patients have cancer, are older, have nutritional problems, comorbid conditions and some have undergone radiation and chemotherapy with possible depressed immunity. ${ }^{42}$ To date there is insufficient information on the effect of COVID-19 in cancer patients.

No reported series of patients who underwent emergency head and neck surgery exist till date. Hence, one must extrapolate from the experience in other areas to develop a strategy for patients with head and neck cancer requiring emergency surgery. According to Chen et al. ${ }^{43}$, the National Health Commission of China recommends collecting nasopharyngeal swab samples to test for COVID-19 and have a chest computed tomography $(\mathrm{CT})$ for all pregnant infected patients. Patients must be transferred between the isolation ward and the OR by a negative pressure isolation transfer cabin. All the personnel involved must wear level 3 protective medical equipment (BSL-3). A negative pressure operating room must be used and its preparation and personal protection include the use of BSL-3 (N95 masks, goggles, protective suits, disposable medical caps and rubber gloves). Medical personnel should enter and exit the operating room in accordance with the principles of clean area, contaminated pollution area, and two buffer zones. Designated nurses must ensure the implementation of standard procedures. ${ }^{43}$ For those who receive general anesthesia, endotracheal intubation and PAPR are mandatory. ${ }^{44}$

Chen et al. ${ }^{43}$ used rapid inhalation ( 2 minutes) induction of general anesthesia ( $8 \%$ sevoflurane in $100 \%$ oxygen) followed by intravenous injections of $2 \%$ lidocaine $(1-1.5 \mathrm{mg} / \mathrm{kg})$, remifentanil $(1-2 \mathrm{mg} / \mathrm{kg})$ and succinylcholine choline $(1-2 \mathrm{mg} / \mathrm{kg})$ to ensure optimal intubating conditions. All the patients included in their study were parturients and sevoflurane was used to maintain anesthesia before delivery, with sufentanil (0.25$0.35 \mu \mathrm{g} / \mathrm{kg})$ and an infusion of propofol $(50-100 \mu \mathrm{g} / \mathrm{kg} / \mathrm{min})$ used to maintain anesthesia after delivery.

After the surgical procedure, the anesthesia workstation was disinfected for two hours with an anesthesia circuit sterilizer (containing $12 \%$ hydrogen peroxide). Chlorine-containing disinfectant $(2,000 \mathrm{mg} / \mathrm{L})$ was used to clean the OR floor and wipe the surface of all reusable medical equipment. All medical devices, such as surgical instruments, were soaked for $30 \mathrm{~min}$ in $2,000 \mathrm{mg} / \mathrm{L}$ chlorine-containing disinfectant, then sealed and collected into double-layer disposable medical waste bags in the cleaning room and sent to the designated disinfection area. After the OR was cleaned, the air purification system was shut down after 30 min of continuous operation of negative pressure laminar flow. Then, an ultra-low volume of $3 \%$ hydrogen peroxide $(20-30 \mathrm{~mL} / \mathrm{m})$ was used to closed fumigate the OR for two hours. Finally, the negative pressure ventilation of the OR was turned on again. All medical staff who were involved in surgical procedure were required to have a SARS-CoV-2 virus detection test (RT-PCR of nasopharyngeal swabs) and CT scans once 
every two weeks. $4^{43}$

Surgeons performing endoscopic sinus surgery seem to be at particular risk due to the high concentration of viral particles in the nasopharyngeal region. The most recent Stanford University guidelines advise canceling elective cases. For COVID-positive patients that cannot be rescheduled, use powered air-purifying respirators (PAPR) if at all possible. ${ }^{16}$

\section{Conclusions}

The world is experiencing an unprecedented challenge during the COVID-19 pandemic. Lessons learned include the need to "flatten the curve" and prevent spread in the community; increased risks of transmission to otolaryngologists, oral maxillofacial surgeons, dentists, and anesthesiologists; and tactics to limit the risk of spread. Rigorous adherence to infection control measures and attention to rapidly changing policies and procedures is essential to mitigate the spread of this disease. New challenges are emerging, including the ability provide care for patients with an urgent need for time-sensitive care, such as head and neck cancer patients, when our healthcare systems are at running capacity and/or overwhelmed.

This review summarizes some of the more readily available clinical protocols for head and neck specialists caring for patients in an environment of a SARS CoV-2 mediated COVID-19 pandemic. Recommendations are based largely on relatively small series, often from single centers, and national position statements. Some represent expert opinion and application from experiences with other diseases. Resources and policies may vary not only between countries, but also between hospitals.

The medical community is accruing information rapidly and standards are likely to change quickly with additional results. We must learn from one another as the disease crosses the globe so as to integrate the lessons into practice before too many patients and their caregivers succumb in the pandemic. Only time will reveal which approaches are most robust and generally applicable. Head and neck specialists should not allow their commitment to patient care override self-preservation-healthcare personnel form the most valuable resource in every country during the pandemic. There is a need for system and hospital-based decision-making to support healthcare teams, which are composed of dedicated but vulnerable individuals.

\section{References}

1. Meng L, Hua F, Bian Z. Coronavirus Disease 2019 (COVID-19): Emerging and Future Challenges for Dental and Oral Medicine. J Dent Res 2020:22034520914246.doi:10.1177/0022034520914246

2. Chan KW, Wong VT, Tang SCW. COVID-19: An Update on the Epidemiological, Clinical, Preventive and Therapeutic Evidence and Guidelines of Integrative Chinese-Western Medicine for the Management of 2019 Novel Coronavirus Disease. Am J Chin Med 2020:1-26.doi:10.1142/S0192415X20500378

3. Chen J, Qi T, Liu L, et al. Clinical progression of patients with COVID-19 in Shanghai, China. J Infect 2020.doi:10.1016/j.jinf.2020.03.004

4. $\quad \mathrm{Wu} \mathrm{Z}, \mathrm{McGoogan}$ JM. Characteristics of and Important Lessons From the Coronavirus Disease 2019 (COVID-19) Outbreak in China: Summary of a Report of 72314 Cases From the Chinese Center for Disease Control and Prevention. JAMA 2020.doi:10.1001/jama.2020.2648

5. Zhou F, Yu T, Du R, et al. Clinical course and risk factors for mortality of adult inpatients with COVID-19 in Wuhan, China: a retrospective cohort study. Lancet 2020.doi:10.1016/S0140-6736(20)30566-3

6. Liang W, Guan W, Chen R, et al. Cancer patients in SARS-CoV-2 infection: a nationwide analysis in China. Lancet Oncol 2020;21:335-337.doi:10.1016/S1470-2045(20)30096-6

7. Wang H, Zhang L. Risk of COVID-19 for patients with cancer. Lancet Oncol 2020.doi:10.1016/S14702045(20)30149-2

8. Chan Y, Wong W, Lam W. Practical Aspects of Otolaryngologic Clinical Services During the 2019 Novel Coronavirus Epidemic. An Experience in Hong Kong. JAMA Otolaryngology-Head \& Neck Surgery 
2020.doi:10.1001/jamaoto.2020.0488

9. Wang J, Zhou M, Liu F. Exploring the reasons for healthcare workers infected with novel coronavirus disease 2019 (COVID-19) in China. J Hosp Infect 2020.doi:10.1016/j.jhin.2020.03.002

10. Remuzzi A, Remuzzi G. COVID-19 and Italy: what next? Lancet 2020.doi:10.1016/S0140$6736(20) 30627-9$

11. Koh D. Occupational risks for COVID-19 infection. Occup Med (Lond) 2020;70:3-5.doi:10.1093/occmed/kqaa036

12. Ran L, Chen X, Wang Y, Wu W, Zhang L, Tan X. Risk Factors of Healthcare Workers with Corona Virus Disease 2019: A Retrospective Cohort Study in a Designated Hospital of Wuhan in China. Clin Infect Dis 2020.doi:10.1093/cid/ciaa287

13. Lai THT, Tang EWH, Chau SKY, Fung KSC, Li KKW. Stepping up infection control measures in ophthalmology during the novel coronavirus outbreak: an experience from Hong Kong. Graefes Arch Clin Exp Ophthalmol 2020.doi:10.1007/s00417-020-04641-8

14. Lu D, Wang H, Yu R, Yang H, Zhao Y. Integrated infection control strategy to minimize nosocomial infection of coronavirus disease 2019 among ENT healthcare workers. J Hosp Infect 2020.doi:10.1016/j.jhin.2020.02.018

15. Joob B, Wiwanitkit V. COVID-19 in medical personnel: observation from Thailand. J Hosp Infect 2020.doi:10.1016/j.jhin.2020.02.016

16. Patel Z, Fernandez-Miranda J, Hwang P, et al. Precautions for endoscopic transnasal skull base surgeryduring the covid-19 pandemic. 202024 March. Available from: https://www.entnet.org/sites/default/files/uploads/cor 19_endosb_lettertoeditor_neurosurgery_update3.23.20.pdf

17. Rimmer A. Covid-19: What's the current advice for UK doctors? BMJ 2020;368:m978.doi:10.1136/bmj.m978

18. Schwartz J, King CC, Yen MY. Protecting Health Care Workers during the COVID-19 Coronavirus Outbreak -Lessons from Taiwan's SARS response. Clin Infect Dis 2020.doi:10.1093/cid/ciaa255

19. Ng K, Poon BH, Kiat Puar TH, et al. COVID-19 and the Risk to Health Care Workers: A Case Report. Ann Intern Med 2020.doi:10.7326/L20-0175

20. Maxwell DN, Perl TM, Cutrell JB. "The Art of War" in the Era of Coronavirus Disease 2019 (COVID-19). Clin Infect Dis 2020.doi:10.1093/cid/ciaa229

21. Li T. Diagnosis and clinical management of severe acute respiratory syndrome Coronavirus 2 (SARSCoV-2) infection: an operational recommendation of Peking Union Medical College Hospital (V2.0). Emerg Microbes Infect 2020;9:582-585.doi:10.1080/22221751.2020.1735265

22. Schwartz DA. An Analysis of 38 Pregnant Women with COVID-19, Their Newborn Infants, and Maternal-Fetal Transmission of SARS-CoV-2: Maternal Coronavirus Infections and Pregnancy Outcomes. Arch Pathol Lab Med 2020.doi:10.5858/arpa.2020-0901-SA

23. Li Y, Zhao R, Zheng S, et al. Lack of Vertical Transmission of Severe Acute Respiratory Syndrome Coronavirus 2, China. Emerg Infect Dis 2020;26.doi:10.3201/eid2606.200287

24. Huh S. How to train the health personnel for protecting themselves from novel coronavirus (COVID-19) infection during their patient or suspected case care. J Educ Eval Health Prof 2020;17:10.doi:10.3352/jeehp.2020.17.10

25. Asmundson GJG, Taylor S. How health anxiety influences responses to viral outbreaks like COVID19: What all decision-makers, health authorities, and health care professionals need to know. J Anxiety Disord 2020;71:102211.doi:10.1016/j.janxdis.2020.102211

26. Ioannidis JPA. Coronavirus disease 2019: the harms of exaggerated information and non-evidencebased measures. Eur J Clin Invest 2020:e13222.doi:10.1111/eci.13222 
27. Asmundson GJG, Taylor S. Coronaphobia: Fear and the 2019-nCoV outbreak. J Anxiety Disord 2020;70:102196.doi:10.1016/j.janxdis.2020.102196

28. Wang W, Xu Y, Gao R, et al. Detection of SARS-CoV-2 in Different Types of Clinical Specimens. Jama 2020.doi:10.1001/jama.2020.3786

29. Zou L, Ruan F, Huang M, et al. SARS-CoV-2 Viral Load in Upper Respiratory Specimens of Infected Patients. N Engl J Med 2020;382:1177-1179.doi:10.1056/NEJMc2001737

30. Hopman J, Allegranzi B, Mehtar S. Managing COVID-19 in Low- and Middle-Income Countries. JAMA 2020.doi:10.1001/jama.2020.4169

31. Li LQ, Huang T, Wang YQ, et al. 2019 novel coronavirus patients' clinical characteristics, discharge rate and fatality rate of meta-analysis. J Med Virol 2020.doi:10.1002/jmv.25757

32. van Doremalen N, Bushmaker T, Morris DH, et al. Aerosol and Surface Stability of SARS-CoV-2 as Compared with SARS-CoV-1. N Engl J Med 2020.doi:10.1056/NEJMc2004973

33. Centers for Disease Control and Prevention. Interim U.S. Guidance for Risk Assessment and Public Health Management of Healthcare Personnel with Potential Exposure in a Healthcare Setting to Patients with Coronavirus Disease (COVID-19). 2020 March 22. Available from: https://www.cdc.gov/coronavirus/2019ncov/hcp/guidance-risk-assesment-hcp.html

34. Tikka T, Kavanagh K, Lowit A, et al. Head and neck cancer risk calculator (HaNC-RC)-V.2. Adjustments and addition of symptoms and social history factors. Clin Otolaryngol 2020.doi:10.1111/coa.13511

35. Hardman J, Tikka T, on behalf of INTEGRATE (The UK ENT Trainee Research Network). United Kingdom Head and Neck Cancer Surveillance study 2018. In: British Association of Head \& Neck Oncologists (BAHNO). London; 2019.

36. Zuo M, Huang Y, Ma W, et al. Expert Recommendations for Tracheal Intubation in Critically ill Patients with Noval Coronavirus Disease 2019. Chin Med Sci J 2020.doi:10.24920/003724

37. Aminnejad R, Salimi A, Saeidi M. Lidocaine during intubation and extubation in patients with coronavirus disease (COVID-19). Can J Anaesth 2020.doi:10.1007/s12630-020-01627-2

38. Yang SS, Wang NN, Postonogova T, et al. Intravenous lidocaine to prevent postoperative airway complications in adults: a systematic review and meta-analysis. Br J Anaesth 2020;124:314-323.doi:10.1016/j.bja.2019.11.033

39. Wong J, Goh QY, Tan Z, et al. Preparing for a COVID-19 pandemic: a review of operating room outbreak response measures in a large tertiary hospital in Singapore. Can J Anaesth 2020.doi:10.1007/s12630020-01620-9

40. Ti LK, Ang LS, Foong TW, Ng BSW. What we do when a COVID-19 patient needs an operation: operating room preparation and guidance. Can J Anaesth 2020.doi:10.1007/s12630-020-01617-4

41. American Academy of Otolaryngology Head and Neck Surgery (AAO-HNS). New Recommendations Regarding Urgent and Nonurgent Patient Care. 2020 March 22. Available from: https://www.entnet.org/content/newrecommendations-regarding-urgent-and-nonurgent-patient-care

42. Schreiber RD, Old LJ, Smyth MJ. Cancer immunoediting: integrating immunity's roles in cancer suppression and promotion. Science 2011;331:1565-70.doi:10.1126/science.1203486

43. Chen R, Zhang Y, Huang L, Cheng BH, Xia ZY, Meng QT. Safety and efficacy of different anesthetic regimens for parturients with COVID-19 undergoing Cesarean delivery: a case series of 17 patients. Can J Anaesth 2020.doi:10.1007/s12630-020-01630-7

44. Wax RS, Christian MD. Practical recommendations for critical care and anesthesiology teams caring for novel coronavirus (2019-nCoV) patients. Can J Anaesth 2020.doi:10.1007/s12630-020-01591-x 